\title{
A Laboratory of Stellar Nuleosynthesis: Isotope Ratios in the Magellanic Clouds
}

\author{
Yi-nan Chin \\ Academia Sinica Institute of Astronomy \& Astrophysics, PO Box 1-87 \\ Nankang, 11529 Taipei, Taiwan
}

\begin{abstract}
In radio astronomy, interstellar isotope ratios have been measured for more than two decades towards different parts of the Milky Way and central regions of some star-burst galaxies. While signals are often too weak to detect rare isotopic species in relatively distant extragalactic sources, our Galaxy only provides an environment with limited metallicity range. Obviously, this constraint can be removed by observing isotopic species in the Magellanic Clouds, located only 50-60 kpc away from us. We thus observed isotope ratios of hydrogen, carbon, nitrogen, oxygen, and sulfur and the results are given in Table 1.
\end{abstract}

\section{Introduction}

It is clear that most of the elements (except of a few which were formed soon after the Big Bang) are synthesized in the interior of stars. As the source of stellar energy, nuclear reactions lead to the formation of a great variety of elements and isotopes. Although there are many reactions and therefore many parameters involved when trying to model stellar interiors, the synthesis of isotopes can be simplified by introducing a concept discriminating between "primary" and "secondary" nuclei. While the "primary" species are synthesized and released by massive stars or by an initial stellar generation, the synthesis of "secondary" nuclei either takes place in low mass stars or in stars that are formed at later times and that possess certain "seed" nuclei. As a consequence, the primary species are released earlier into the interstellar medium than the secondary species. Even this approach may be complex, since some nuclei are synthesized in more than one reaction scheme, possible involving both "primary" and "secondary" formation. Nevertheless, we can observe isotope abundance ratios as a function of metallicity. This allows us to determine how "primary" (or "secondary") a given species is with respect to another, thus providing important constraints on stellar nucleosynthesis and "chemical" evolution.

\section{Observations}

The observations were carried out during several periods between 1997 and 1998 using the 15-m Swedish-ESO Submillimetre Telescope (SEST) at La Silla, Chile. The ${ }^{12} \mathrm{C} /{ }^{13} \mathrm{C}$ ratio has been derived from the line intensity ratio of $\mathrm{HCN}$ to $\mathrm{H}^{13} \mathrm{CN}$. The opacity of the $\mathrm{HCN}(1-0)$ line has been determined by a comparison 
of intensities of its hyperfine components. Similarly, the ${ }^{14} \mathrm{~N} /{ }^{15} \mathrm{~N}$ ratio has been obtained by additional observations of $\mathrm{HC}^{15} \mathrm{~N}$.

The $\mathrm{D} / \mathrm{H}$ ratio has been obtained from the line intensity ratio of $\mathrm{H}^{13} \mathrm{CO}^{+}$ and $\mathrm{DCO}^{+}$, as well as $\mathrm{H}^{13} \mathrm{CN}$ and $\mathrm{DCN}$, by applying the ${ }^{12} \mathrm{C} /{ }^{13} \mathrm{C}$ ratio derived before. Fractionation is estimated applying a detailed astrochemical model.

The oxygen ${ }^{18} \mathrm{O} /{ }^{17} \mathrm{O}$ isotope ratio has been derived from the intensities of the respective isotopic species of the $\mathrm{CO}$ molecule and applying the ${ }^{12} \mathrm{C} /{ }^{13} \mathrm{C}$ ratio from $\mathrm{HCN}$ observation. Similar to the oxygen isotope ratio, the sulfur isotope ratios have been calculated by observing CS isotopic species and applying the ${ }^{12} \mathrm{C} /{ }^{13} \mathrm{C}$ ratio.

\section{Discussion}

In Table 1 we present a brief summary of isotope abundance ratios obtained in the Magellanic Clouds, our Galaxy, and nearby star-burst nuclei. While the $[\mathrm{H} / \mathrm{D}]$ ratio is related to cosmology, other ratios are suitable tracers of stellar nucleosynthesis. The nitrogen and oxygen ratios determined in the LMC (which represents a less evolved environment) suggest some corrections to the stellar nucleosynthesis model. For instance, the very low ${ }^{14} \mathrm{~N} /{ }^{15} \mathrm{~N}$ ratio indicates that ${ }^{15} \mathrm{~N}$ has to be synthesized by massive stars. This is consistent with the recent massive star models which take the effects of rotation on the stellar structure and nucleosynthesis into account (Langer et al. 1999).

Table 1. Isotope abundance ratios in various astronomical environments ${ }^{\dagger}$

\begin{tabular}{ccccccc}
\hline Isotope & $\begin{array}{c}\text { Magellanic } \\
\text { Clouds }\end{array}$ & $\begin{array}{c}\text { Galactic } \\
\text { Center }\end{array}$ & $\begin{array}{c}\text { Inner } \\
\text { Disk }\end{array}$ & $\begin{array}{c}\text { Local } \\
\text { ISM }\end{array}$ & $\begin{array}{c}\text { Solar } \\
\text { System }\end{array}$ & $\begin{array}{c}\text { Starburst } \\
\text { Nuclei }\end{array}$ \\
\hline${ }^{12} \mathrm{C} /{ }^{13} \mathrm{C}$ & $62 \pm 5$ & $25 \pm 5$ & $40 \pm 10$ & $70 \pm 10$ & 90 & $\approx 50$ \\
${ }^{14} \mathrm{~N} /{ }^{15} \mathrm{~N}$ & $114 \pm 14$ & $900 \pm 200$ & $375 \pm 60$ & $450 \pm 60$ & 272 & $>>100$ \\
${ }^{16} \mathrm{O} /{ }^{18} \mathrm{O}$ & $>2000$ & $250 \pm 30$ & $300 \pm 30$ & $540 \pm 30$ & 499 & $150-200$ \\
${ }^{18} \mathrm{O} /{ }^{17} \mathrm{O}$ & $1.8 \pm 0.4$ & $3.5 \pm 0.2$ & $3.6 \pm 0.3$ & $3.6 \pm 0.3$ & 5.3 & $>8$ \\
${ }^{32} \mathrm{~S} /{ }^{34} \mathrm{~S}$ & $18 \pm 6$ & - & $24 \pm 5$ & $24 \pm 5$ & 22.6 & - \\
${ }^{34} \mathrm{~S} / /^{33} \mathrm{~S}$ & $3.0 \pm 0.7$ & - & $6 \pm 1$ & $6 \pm 1$ & 5.6 & - \\
\hline \hline
\end{tabular}

\footnotetext{
${ }^{\dagger}$ While isotope abundance ratios of hydrogen, carbon, and oxygen are summarized by Wilson \& Rood (1994), those of sulfur are measured by Chin et al. (1996).

${ }^{\ddagger}$ The values of the Solar System represent the isotope abundance ratios in the LISM $4.5 \times 10^{9}$ years ago.
}

\section{References}

Chin, Y.-N., Henkel, C., Whiteoak, J.B., Langer, N., \& Churchwell, E.B. 1996, A\&A, 305, 960

Langer, N., Heger, A., \& García-Segura, G. 1999, RvMA, 11, 57

Wilson, T.L., \& Rood, T.R. 1994, ARA\&A, 32, 191 


\section{Discussion}

George Sonneborn: How did you derive the $\mathrm{D} / \mathrm{H}$ value of $2 \times 10^{-5}$ ?

Chin: By observing the deuterated molecules $\mathrm{DCO}^{+}$and DCN. However, some assumptions (such as the same excitation condition) have to be made and, with a model simulation of chemical reaction, we can derive the $\mathrm{D} / \mathrm{H}$ ratios. They are consistent toward different locations as well as by different molecular species. Conservatively, I would suggest this ratio to be $2-4 \times 10^{-5}$ in the LMC. 